\title{
Passive Linearization of the Magnetic Bunch Compression Using Self-Induced Fields
}

\author{
G. Penco, ${ }^{1,}{ }^{*}$ E. Allaria, ${ }^{1}$ I. Cudin, ${ }^{1}$ S. Di Mitri, ${ }^{1}$ D. Gauthier, ${ }^{2}$ S. Spampinati, ${ }^{1}$ M. Trovó, ${ }^{1}$ \\ L. Giannessi, ${ }^{1,3}$ E. Roussel, ${ }^{1,4}$ S. Bettoni, ${ }^{5}$ P. Craievich, ${ }^{5}$ and E. Ferrari ${ }^{5}$ \\ ${ }^{1}$ Elettra-Sincrotrone Trieste, Area Science Park, 34149 Trieste, Italy \\ ${ }^{2}$ LIDYL, CEA, CNRS, Universit Paris-Saclay, CEA Saclay, 91191 Gif-sur-Yvette, France \\ ${ }^{3}$ ENEA C.R. Frascati, Via E. Fermi 45, 00044 Frascati (Roma), Italy \\ ${ }^{4}$ Laboratoire PhLAM, UMR CNRS 8523, Université Lille 1, Sciences et Technologies, 59655 Villeneuve d'Ascq, France \\ ${ }^{5}$ Paul Scherrer Institut, $\mathrm{CH}-5232$ Villigen PSI, Switzerland
}

(Received 4 July 2017; published 1 November 2017)

\begin{abstract}
In linac-driven free-electron lasers, colliders, and energy recovery linacs, a common way to compress the electron bunch to kiloampere level is based upon the implementation of a magnetic dispersive element that converts particle energy deviation into a path-length difference. Nonlinearities of such a process are usually compensated by enabling a high harmonic rf structure properly tuned in amplitude and phase. This approach is however not straightforward, e.g., in $C$-band and $X$-band linacs. In this Letter we demonstrate that the longitudinal self-induced field excited by the electron beam itself is able to linearize the compression process without any use of high harmonic rf structure. The method is implemented at the FERMI linac, with the resulting high quality beam used to drive the seeded free-electron laser during user experiments.
\end{abstract}

DOI: 10.1103/PhysRevLett.119.184802

Most high-gain free-electron lasers (FELs) operating in the vacuum ultraviolet [1-3] and $\mathrm{x}$-ray [4,5] spectral range rely on a high-brightness electron beam accelerated in a linac, with an kiloampere peak current, possibly uniform over a core bunch from a few to hundreds of femtoseconds. To achieve such a high peak current, the electron bunch generated in the injector needs to be compressed by 1 or more orders of magnitude. A common way to compress the electron bunch consists in imposing a time-energy correlation to the electrons before injecting them in a dispersive magnetic insertion (e.g., a chicane) where the path length is energy dependent. The time-energy correlation is induced by setting off crest the radio-frequency (rf) phase of selected accelerating structures and in the chicane the time-energy correlation is converted by the dispersion into a compression of the temporal distribution. High order terms both in the time-energy distribution induced by the curvature of the rf accelerating field and in the chicane path length versus beam energy translate into a nonlinear longitudinal compression. These higher order terms are usually compensated by a short active rf accelerating structure at a higher harmonic $(\mathrm{HH})$ of the main accelerating linac [6,7], typically the 3rd or 4th harmonic. Other theoretical methods are based upon the implementation of quadrupoles and sextupoles to manipulate the second order longitudinal dispersion in the bunch compressor [8-12]. However, these schemes typically suffer from residual chromatic aberrations that degrade the final beam transverse emittance. Without linearizing the bunch compression, the electron longitudinal phase space is characterized by a nonuniform current density with a current peak at the head and a steep slope along the bunch. The region of the current peak is also characterized by a large energy spread which is detrimental for the FEL amplification and is almost incompatible with the operation of a seeded FEL where the quality and uniformity of the longitudinal phase space is one of the most important prerequisites to preserve the spectral quality of the light.

Despite all existing FELs operating successfully with a $\mathrm{HH}$ structure, alternative methods have been considered to avoid the implementation of an active rf system. Options lacking a $\mathrm{HH}$ structure are particular appealing in the case of new concept machines based upon an $X$-band linac [13] that would require high frequency harmonic if sections in the $K_{a}$ or $V$ band, increasing the overall system complexity.

An alternative was proposed in 2010 [14] consisting of a passive dielectric-lined waveguide (DLW) designed to excite longitudinal wakefields that should provide the needed positive time-energy curvature to compensate the nonlinearities. In the last years, several facilities have developed passive devices as thin dielectric layers, resistive pipes of small radius, and dielectric corrugated pipes to remove the undesirable residual linear time-energy correlation in the bunch before entering the undulators [15-17], or to linearize the longitudinal phase space of the uncompressed beam $[18,19]$, or to shape the current distribution and obtain a linearly ramped temporal profile for injection into a dielectric wakefield accelerator [20], or finally to tailor an electron bunch with a temporal-transverse correlation to produce multicolor x-ray FEL pulses [21]. However, the original concept [14] to use a self-induced field to passively linearize 
the compression process without any $\mathrm{HH}$ cavities has never been proven before.

Intrinsic geometrical longitudinal wakefields, generated by the beam in the accelerating sections, may become relevant in the case of short bunches and/or small inner iris rf sections. In a multistage compression scheme, the amplitude and curvature of these wakefields may be tuned by shaping the beam profile after one compressor to reach the proper time-energy curvature compensating the nonlinearities after the subsequent compressor. This applies even for a hybrid compression scheme, where the firststage is realized by exploiting the velocity bunching method [22].

In this Letter we present two experiments carried out at the accelerating infrastructure driving the FERMI seeded free electron lasers [23], where we demonstrated the linearization of the compression process without using any active $\mathrm{HH}$ structure.

The FERMI linac layout is shown in Fig. 1.

In both experiments the double-stage compressor scheme has been adopted, and the first compressor (BC1) serves to tune the bunch length in order to generate sufficiently strong wakefields downstream to linearize the compression in the second compressor (BC2). In the first experiment we exploit the fundamental contribution to the longitudinal dynamics of the wakefields in the rf sections after $\mathrm{BC} 1$ and before $\mathrm{BC} 2$, i.e., L02 and L03, to obtain a uniform current distribution. The achieved quality of the beam was comparable to the one obtainable with the $\mathrm{HH}$ structure linearization system and the setup was used to drive the FEL during pilot user experiments.

In the second experiment, we add a DLW passive element after $\mathrm{BC} 1$ and we exploit the combined effect of the wakefields induced in the DLW itself and in the L02 and L03 linacs.

The FERMI linac [24] is composed of two types of $S$-band rf accelerating structures. The beam is accelerated by a sequence of traveling wave (TW) structures in the first part of the linac, and by backward-traveling wave (BTW) structures, characterized by a small iris ( $5 \mathrm{~mm}$ radius) and substantially higher impedance [25], in the second. The excited longitudinal wakefields strongly affect the longitudinal phase space in this second type of structures, inducing a positive curvature that in standard configuration

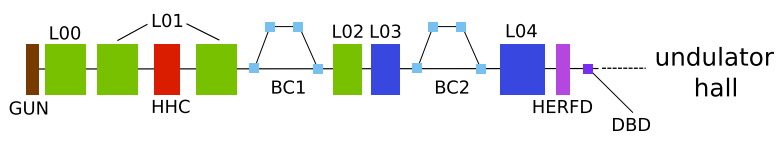

FIG. 1. FERMI linac layout including the rf photocathode gun, linacs L00, L01, L02 (SLAC-type traveling wave accelerating structures), L03 and L04 (high-impedance BTW accelerating structures), the high harmonic cavity (i.e., $X$-band rf section), and the two magnetic bunch compressors $\mathrm{BC} 1$ and $\mathrm{BC} 2$. The high energy deflecting cavity (HERFD) and the diagnostic beam dump (DBD) are placed after L04. is removable only by shaping the temporal profile at the photoinjector [26]. The result of this work is to exploit this effect to control the nonlinearity originating in the compression process. The scheme however is not specific to the FERMI linac layout. Even in a linac based on moderate impedance accelerating structures, an element with high impedance, such as dielectric-lined or corrugated waveguides, can be implemented to achieve and control the self-induced fields on the beam itself, in superposition on or substitution for an active $\mathrm{HH}$ cavity.

Analytical expressions of the wake function for the FERMI BTW sections were found numerically and we refer to Ref. [25] for further details. The convolution of the wake function with the bunch temporal profile compressed in $\mathrm{BC} 1$ provides the wake potential interacting with the bunch itself. Therefore, the compression factor set in $\mathrm{BC} 1$ becomes a free parameter to tune the effect of the longitudinal wakefields generated in L02 and L03. This parameter should be optimized to compensate the negative rf curvature imposed in L01, L02, and L03, and the negative energy-time curvature of the second order terms of both chicanes. In the standard configuration adopting the $\mathrm{HH}$ cavity, the required positive curvature is provided by phasing the $\mathrm{rf}$ in the decelerating mode and tuning the harmonic voltage $V_{h}$ to [6]

$$
e V_{h}=\frac{E_{0}\left(1+\frac{1}{2 \pi^{2}} \frac{\lambda_{s}^{2} T_{566}}{\left|R_{56}\right|^{3}}(1-1 / \mathrm{CF})^{3}\right)-E_{i}}{\left(\lambda_{s} / \lambda_{h}\right)^{2}-1},
$$

where $e$ is the electron charge, $E_{i}$ and $E_{0}$ are, respectively, the beam energy before the accelerating sections and at the chicane, CF is the compression factor, $\lambda_{s}$ and $\lambda_{h}$ are, respectively, the rf wavelength of the main system and of the HH cavity. The terms $R_{56}$ and $T_{566}$ are the linear and the second order term of the chicane time-energy dependence. From Eq. (1) it follows that decreasing the energy of the beam at the bunch compressor relaxes the $V_{h}$ amplitude needed to linearize the compression, and the same is true even for the required longitudinal wake potential. On the other side compressing the beam at low energy increases the effects of the space charge forces and microbunching instabilities so it is necessary to find a trade-off. In the light of these considerations we have set the FERMI linac beam energy in $\mathrm{BC} 1$ at about $190 \mathrm{MeV}$ and in $\mathrm{BC} 2$ at about $520 \mathrm{MeV}$. We have tuned the linac in the nominal conditions used to operate the FERMI FELs, with a charge per bunch of $700 \mathrm{pC}$ and a peak current at the injector of about 70 A [27]. We aimed to compress the beam to produce the flat current distribution in the bunch core optimizing the seeding process. The LITRACK code [28] has been used to identify the best configuration capable of producing a uniform current distribution at the end of the linac and the results are reported in Fig. 2. Without using the $\mathrm{HH}$ cavity, the beam temporal profile after $\mathrm{BC} 1$, set with a moderate compression of about a factor 4 , presents a 

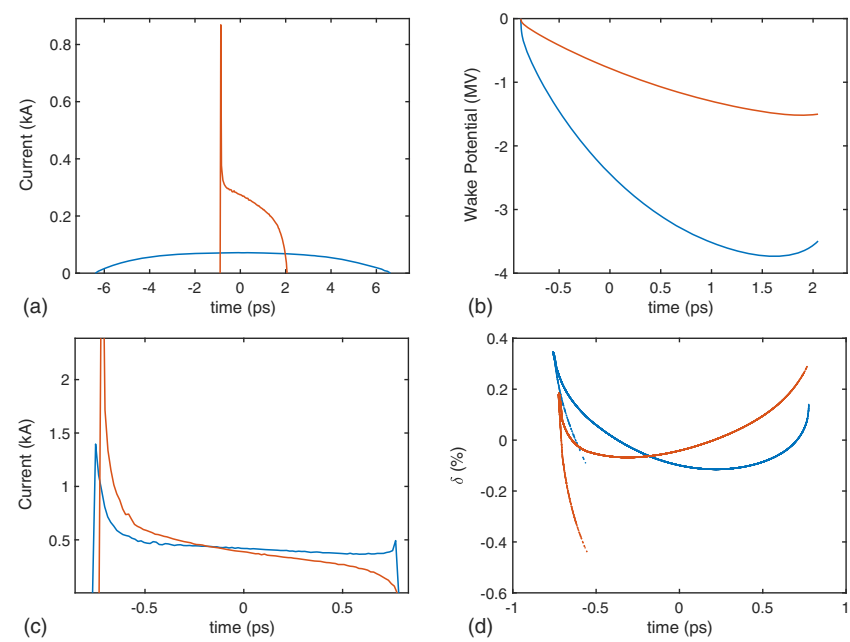

FIG. 2. LITRACK simulation results. (a) Current profile of a $700 \mathrm{pC}$ bunch at the FERMI injector exit at about $100 \mathrm{MeV}$ (blue line) and after $\mathrm{BC} 1$ (red line), which is set with $R_{56, \mathrm{BC} 1}=-41 \mathrm{~mm}$. (b) The wake potential in L03 induced by the electron bunch compressed in $\mathrm{BC} 1$ when $\mathrm{L} 03$ is composed of BTW sections (blue) or TW SLAC-type sections (red). Current distribution (c) and longitudinal phase space (d) at the end of the linac obtained with (blue line) and without (red line) inclusion of the longitudinal wakefields in the L02 and L03 sections. The head of the bunch is on the left.

high peak current on the head and a steep slope towards the tail [see Fig. 2(a)]. The wake potential induced in L03, composed of BTW sections, has been calculated and compared to a hypothetical case where the L03 was composed of TW SLAC-type structure, reporting the results in Fig. 2(b): the BTW sections are able to excite a wake potential almost a factor 3 larger than the SLACtype accelerating structures.

$\mathrm{BC} 2$ has been set with $R_{56, \mathrm{BC} 2}=-20 \mathrm{~mm}$ and Fig. 2(c) shows the current distribution at the end of the linac simulated by LITRACK considering the actual layout, i.e., L03 composed of BTW sections (blue solid line), and the hypothetical case in which no longitudinal wakefields are excited either in L02 or in L03 (red dotted line). In the case of negligible longitudinal wakefield effects, as, for instance, in case of superconducting accelerating sections, the strong peak current on the head and the steep slope along the bunch are unavoidable without using an active $\mathrm{HH}$ system.

Several machine configurations previously studied with LITRACK were implemented on the FERMI linac and measurements have been carried out to verify the linearization of the compression process without activating the HH cavity. The electron longitudinal phase space has been measured at the end of the linac by using the high energy rf deflector [29] installed after L04 and the beam energy spectrometer that delivers the beam to the diagnostic beam dump (DBD). The vertical deflector imposes a vertical displacement correlated with the electrons' time of arrival, while the spectrometer disperses the particles horizontally
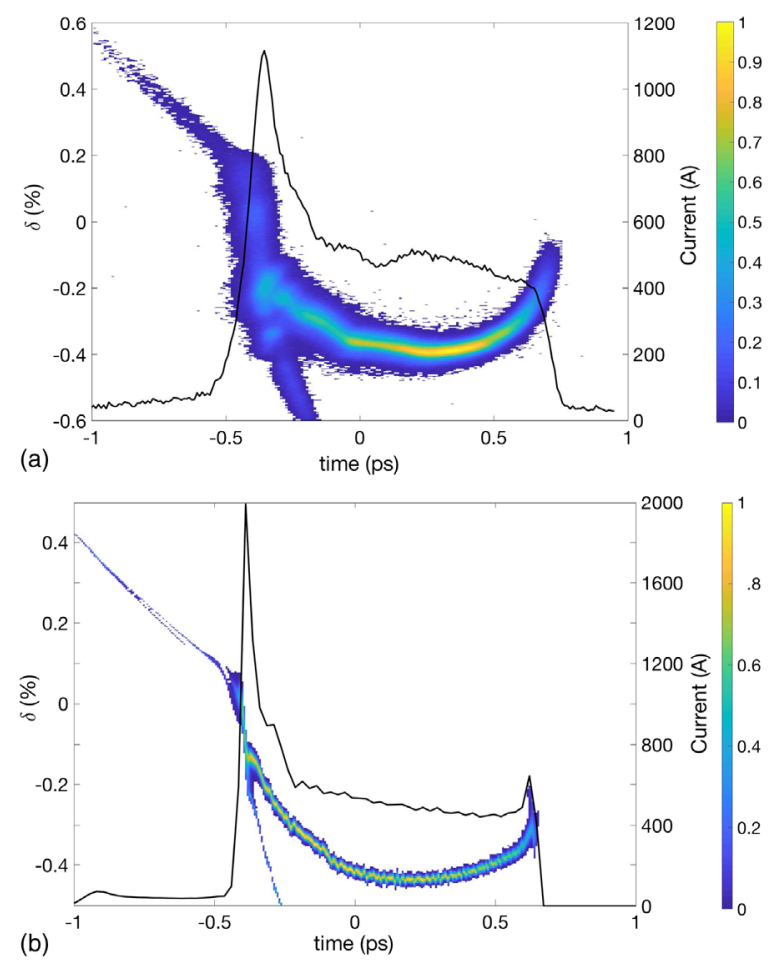

FIG. 3. (a) Longitudinal phase space of a 700-pC electron bunch measured by stretching the electron beam with the rf deflector and energy dispersing it with the DBD spectrometer. (b) Elegant simulation results in the same condition. The head of the bunch is on the left.

according to their energy. The beam temporally stretched in the vertical direction and dispersed in energy in the horizontal direction are then intercepted by an yttriumaluminum-garnet screen in the DBD line where the electron longitudinal phase space is imaged. Figure 3 reports the electron longitudinal phase space measured in an optimized configuration and the corresponding Elegant simulation [30]. The core of the bunch is flat in current and in energy, resulting in a configuration very similar to the nominal FERMI configuration, i.e., enabling the $\mathrm{HH}$ cavity and adopting the single-stage compression [26]. Elegant results are in good agreement with the experiment, reproducing the details of the longitudinal phase space, as the bifurcation due to the strong space charge forces acting on the bunch head electrons. Here, in fact, the space charge increases the energy spread of electrons that are temporally dispersed in the $\mathrm{BC} 2$ chicane, creating a charge distribution in front of the bunch.

This electron bunch, generated in double-stage compression and without the $\mathrm{HH}$ cavity, has been driven along the FEL-1 line to lase in the high gain harmonic generation mode [31], obtaining an intensity and a spectral purity comparable to the ones characterizing FERMI in the nominal conditions. Figure 4 shows the single-shot FEL spectrum acquired at $25.9 \mathrm{~nm}$ : a relative bandwidth of about $3.4 \times 10^{-4}$ has been measured that is close to the 


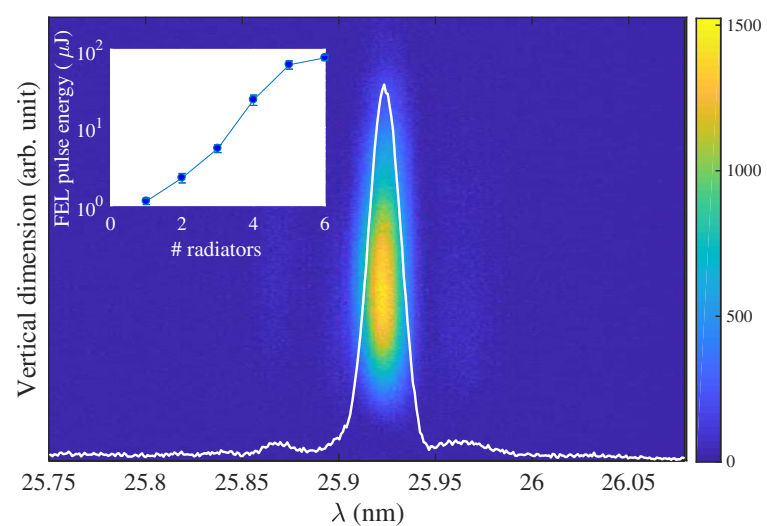

FIG. 4. Single-shot FEL spectrum at $25.9 \mathrm{~nm}$ obtained in the optimized configuration. The inset shows the FEL gain curve at $25 \mathrm{~nm}$ measured progressively detuning each radiator undulator.

Fourier limit and consistent with the typical FERMI output [32]. The exponential growth of the output intensity versus the number of resonant radiators has been measured by progressively detuning each radiator undulator (see the inset of Fig. 4): the FEL reaches the saturation after six undulators with an energy per pulse of about $80 \mu \mathrm{J}$. Although we observed a stronger microbunching instability in a two-stage bunch compression scheme than in the single stage, as already described in Ref. [33], the increase in the uncorrelated energy spread does not affect significantly the FEL performance.

Stimulated by this successful test, we have also considered the original concept of installing a passive DLW after BC1. For this scope, we have implemented the DLW that was built by the Paul Scherrer Institut (PSI) and had been already tested as a passive streaker for time-resolved longitudinal phase space measurements [34]. The PSI DLW is an alumina pipe, $28.5 \mathrm{~mm}$ long, with an external $20 \mu \mathrm{m}$ copper coating. The inner and outer radius are, respectively, 1.65 and $2.4 \mathrm{~mm}$. The fundamental mode excited in the DLW has a frequency of $32 \mathrm{GHz}$, whereas the FERMI active $\mathrm{HH}$ structure has a working frequency at about $12 \mathrm{GHz}$. According to Eq. (1) we need to excite a longitudinal wakefield with an amplitude about 6 times smaller than the required $\mathrm{HH}$ voltage. The 10-m-long diagnostic section after $\mathrm{BC} 1$ [35] and before the L02 linac is equipped by several multiscreen diagnostics, steerer magnets, beam position monitors, and emittance measurement systems, an ideal place to test and characterize the DLW effects on the beam. Moreover, the typical beam spot size in this area is about $100 \mu \mathrm{m}$ or less [36], making it well suited for passing through the DLW small iris. As in the previous experiment, we have used the same nominal bunch of $700 \mathrm{pC}$, with the goal to reach a peak current of about $500 \mathrm{~A}$. After a careful prealignment of this device on the beam axis, we have optimized the electron trajectory to minimize the transversal wakefield effects by looking at the downstream screen and by minimizing the transverse spot size. The projected transverse beam emittance has been measured both in $\mathrm{BC} 1$ and at the end of the linac, obtaining about $1.5 \mathrm{~mm} \mathrm{mrad}$ on both planes (normalized). These values are similar to the nominal ones routinely obtained with the HH cavity.

LiTrACK simulations of the wake potential induced in the DLW and acting on the beam as a function of the compression in $\mathrm{BC} 1$ are plotted in Fig. 5(b). In the case of strong compression [green line in Fig. 5(a)] the induced voltage has an absolute maximum value of about 1.2 MV [green line in Fig. 5(b)]. In the nominal condition the FERMI HH structure voltage is about $17 \mathrm{MeV}$, so according to Eq. (1) it is necessary to induce $\approx 2.8 \mathrm{MeV}$ in the DLW to have an equivalent effect. The longitudinal wakefield potential excited in the DLW added to the one excited in the downstream rf linac sections lowers the required DLW wake potential necessary to flatten the beam current profile. Moreover, the presence of the DLW changes the energy distribution at the entrance of $\mathrm{BC} 2$ and consequently slightly modifies the compression setting in the second stage. In summary, the implementation of the DLW leads to different settings of the compression parameters in both $\mathrm{BC} 1$ and $\mathrm{BC} 2$ with respect to the experiments described above. With the goal to preserve the final bunch length, we have changed the compression ratio between the two stages, by varying the L01 and L02 $\mathrm{rf}$ phase $\left(\phi_{\mathrm{L} 01}\right.$ and $\left.\phi_{\mathrm{L} 02}\right)$, to tune the compression factors in $\mathrm{BC} 1$ and $\mathrm{BC} 2$, respectively. The chicane angles were maintained constant. The reading of a pyrodetector monitoring the long wavelength coherent emission from the bunch, placed after $\mathrm{BC} 2$, has been used as the target in a feedback loop to measure and keep constant the bunch duration [37]. The electron bunch temporal profile has been measured in several
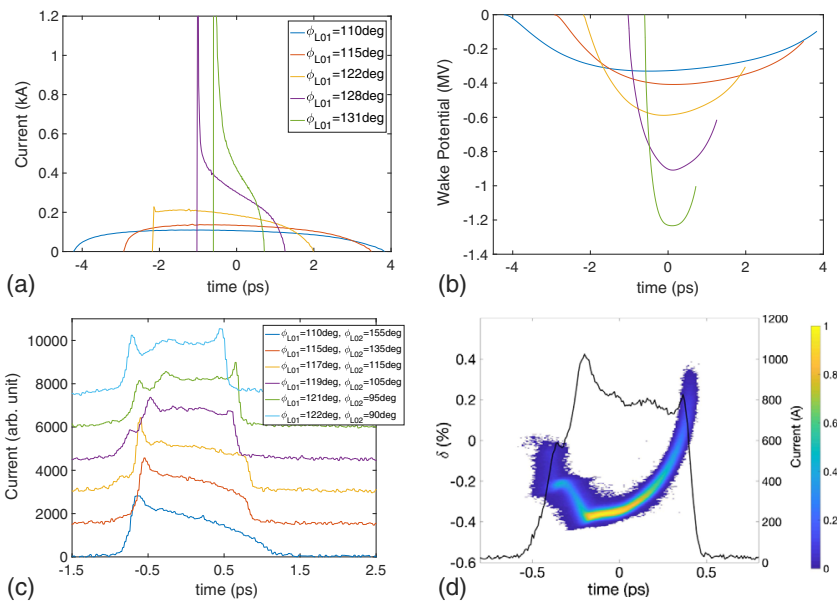

FIG. 5. (a) LITRACK simulated current profiles after BC1 for different compression factors and (b) the corresponding induced voltage in the PSI-DLW device. (c) Beam temporal profiles measured after BC2 as a function of $\phi_{\mathrm{L} 01}$ and $\phi_{\mathrm{L} 02}$, keeping constant the bunch length after BC2. (d) Measured longitudinal phase space of a shorter electron bunch compressed to about $700 \mathrm{~A}$. In all cases the charge per bunch is $700 \mathrm{pC}$ and the head of the bunch is on the left. 
configurations and the results are plotted in Fig. 5(c). The differences are mainly due to the wakefields excited in the DLW and in the L02 and L03 sections: the phase $\phi_{\mathrm{L} 01}$ can be finely tuned to change the compression factor in $\mathrm{BC} 1$ and therefore the self-induced fields in the DLW and in the L02 and L03 sections. A flat current profile in the core is obtained for $\phi_{\mathrm{L} 01}=119 \mathrm{deg}$ and $\phi_{\mathrm{L} 02}=105 \mathrm{deg}$ (considering $90 \mathrm{deg}$ as the on-crest condition). With this scheme, it is also possible to tune the final current profile to a higher level keeping a uniform current distribution in the central core. Figure 5(d) shows the measured electron longitudinal phase space of a $700 \mathrm{pC}$ bunch compressed to about $700 \mathrm{~A}$, obtained without changing the chicane angle and setting $\phi_{\mathrm{L} 01}=121 \mathrm{deg}$ and $\phi_{\mathrm{L} 02}=120 \mathrm{deg}$.

In conclusion, the experiments carried out at FERMI demonstrate that manipulating the self-induced fields passively excited by the beam allows us to linearize the compression process in a magnetic chicane without adopting any rf $\mathrm{HH}$ structure. As a short term perspective this result has triggered the project for a new DLW design, with a tunable aperture, to be installed before $\mathrm{BC} 1$. The added degree of freedom would allow a linear temporal compression in one single stage without any active $\mathrm{HH}$ system to be used as a backup to the existing HH cavity. However, such a design will be particularly attractive for a future linac based upon $C$-band and $X$-band technology, where the realization of $\mathrm{HH}$ structures is much more challenging.

The authors are grateful to the entire FERMI commissioning team for the valuable support in the optimization of the machine during the described experiments.

* giuseppe.penco@elettra.eu

[1] W. Ackermann et al., Nat. Photonics 1, 336 (2007).

[2] E. Allaria et al., Nat. Photonics 6, 699 (2012).

[3] E. Allaria et al., Nat. Photonics 7, 913 (2013).

[4] P. Emma et al., Nat. Photonics 4, 641 (2010).

[5] T. Ishikawa et al., Nat. Photonics 6, 540 (2012).

[6] P. Emma, LCLS Technical Note Report No. SLAC-TN-05004, 2001.

[7] K. Floettmann, T. Limberg, and Ph. Piot, DESY-HH Report No. TESLA-FEL-01-06, 2001.

[8] D. H. Dowell, in Proceedings of the 1997 Particle Accelerator Conference, Vancouver, BC, Canada, 1997 (IEEE, New York, 1997), p. 1888.

[9] P. Piot, D. R. Douglas, and G. A. Krafft, Phys. Rev. ST Accel. Beams 6, 030702 (2003).

[10] Y. Sun, P. Emma, T. Raubenheimer, and J. Wu, Phys. Rev. ST Accel. Beams 17, 110703 (2014).
[11] S. Di Mitri and M. Cornacchia, Europhys. Lett. 109, 62002 (2015).

[12] S. Thorin et al., in Proceedings of the 32nd International Free Electron Laser Conference 2010, Malmö, Sweden (Max-lab.Web) (2010), paper WEPB34.

[13] Y. Sun, C. Adolphsen, C. Limborg-Deprey, T. Raubenheimer, and J. Wu, Phys. Rev. ST Accel. Beams 15, 030703 (2012).

[14] P. Craievich, Phys. Rev. ST Accel. Beams 13, 034401 (2010).

[15] S. Antipov, S. Baturin, C. Jing, M. Fedurin, A. Kanareykin, C. Swinson, P. Schoessow, W. Gai, and A. Zholents, Phys. Rev. Lett. 112, 114801 (2014).

[16] K. L. F. Bane and G. Stupakov, Nucl. Instrum. Methods Phys. Res., Sect. A 690, 106 (2012).

[17] P. Emma, M. Venturini, K. L. F. Bane, G. Stupakov, H. S. Kang, M. S. Chae, J. Hong, C. K. Min, H. Yang, T. Ha, W. W. Lee, C. D. Park, S. J. Park, and I. S. Ko, Phys. Rev. Lett. 112, 034801 (2014).

[18] H. Deng et al., Phys. Rev. Lett. 113, 254802 (2014).

[19] F. Fu et al., Phys. Rev. Lett. 114, 114801 (2015).

[20] G. Andonian, S. Barber, F. H. OShea, M. Fedurin, K. Kusche, C. Swinson, and J. B. Rosenzweig, Phys. Rev. Lett. 118, 054802 (2017).

[21] A. A. Lutman et al., Nat. Photonics 10, 745 (2016).

[22] M. Ferrario et al., Phys. Rev. Lett. 104, 054801 (2010).

[23] E. Allaria et al., J. Synchrotron Radiat. 22, 485 (2015).

[24] S. Di Mitri et al., Nucl. Instrum. Methods Phys. Res., Sect. A 608, 19 (2009).

[25] P. Craievich, T. Weiland, and I. Zagorodnov, Nucl. Instrum. Methods Phys. Res., Sect. A 558, 58 (2006).

[26] G. Penco, M. Danailov, A. Demidovich, E. Allaria, G. De Ninno, S. Di Mitri, W. M. Fawley, E. Ferrari, L. Giannessi, and M. Trovo, Phys. Rev. Lett. 112, 044801 (2014).

[27] G. Penco et al., J. Instrum. 8, P05015 (2013).

[28] K. Bane and P. Emma, Report No. SLAC-PUB-11035, 2005.

[29] P. Craievich et al., IEEE Trans. Nucl. Sci. 62, 210 (2015).

[30] M. Borland, Argonne Report No. APS LS-287, 2000.

[31] L. H. Yu, Phys. Rev. A 44, 5178 (1991).

[32] P. Finetti et al., Phys. Rev. X 7, 021043 (2017).

[33] S. Spampinati et al., Phys. Rev. ST Accel. Beams 17, 120705 (2014).

[34] S. Bettoni, P. Craievich, A. A. Lutman, and M. Pedrozzi, Phys. Rev. Accel. Beams 19, 021304 (2016).

[35] M. Veronose et al., in Proceeding of the 15th Beam Instrumentation Workshop 2012, Newport News, VA USA (2012), paper THAP02.

[36] S. DiMitri, M. Cornacchia, C. Scafuri, and M. Sjostrom, Phys. Rev. ST Accel. Beams 15, 012802 (2012).

[37] M. Ferianis, E. Allaria, E. Ferrari, G. Gaio, G. Penco, F. Rossi, and M. Veronese, High Power Laser Sci. Eng. 4, e13 (2016). 